training program on exercise tolerance, aerobic capacity, and quality of life in patients with systemic lupus erythematosus. Arthritis Rheum 2005;53(6):83844.

Disclosure of Interest: None declared

DOI: 10.1136/annrheumdis-2017-eular.6619

\section{SAT0286 INCIDENCE AND MORTALITY OF PRIMARY SJÖGREN'S SYNDROME: TIME TRENDS OVER A 40-YEAR PERIOD IN A POPULATION-BASED COHORT IN THE UNITED STATES}

G. Maciel ${ }^{1,2}$, C. Crowson ${ }^{3}$, E. Matteson ${ }^{1}$, D. Cornec $4,5 .{ }^{1}$ Rheumatology, Mayo Clinic, Rochester Minnesota, United States; ${ }^{2}$ Hospital Maciel, Montevideo, Uruguay; ${ }^{3}$ Division of Biomedical Statistics and Informatics, Department of Health Sciences Research; ${ }^{4}$ Pulmonary and Critical Care Medicine, Mayo Clinic, Rochester Minnesota, United States; ${ }^{5}$ Rheumatology, CHRU Brest, Brest, France

\section{Background:}

Few studies have reported the incidence of primary Sjögren's syndrome (pSS) in well-defined populations worldwide, and none of them covered a period of time long-enough to analyze potential time trends in incidence rates. Whether pSS is associated with a higher mortality rate compared to the general population is also unclear from previously published studies.

Objectives: To estimate the incidence and mortality rates of pSS among residents of Olmsted County, Minnesota, and their evolution over time.

Methods: All medical records of patients with a diagnosis or suspicion of SS in Olmsted County, MN, from January 1, 2006 to December 31, 2015 were reviewed to identify incident cases of pSS (defined according to physician diagnosis). All patients with doubtful cases and all patients with an associated systemic autoimmune disease were excluded. These cases were combined with a previous 1976-2005 incident cohort from the same population (reference). Incidence rates were age and sex adjusted to the US white 2010 population. Survival rates were compared with the expected rates in the general population of Minnesota.

Results: With 61 incident cases of pSS diagnosed in Olmsted County in 20062015, the total cohort included 172 patients with incident pSS in 1976-2015. Of the 172 patients, $151(88 \%)$ were women and 161 (94\%) were white, with a mean (SD) age at diagnosis of 58.3 (16.7) years. The average age- and sex- adjusted annual incidence for 2006-2015 was 5.9 per 100,000 population (95\% Cl 4.4-7.4), and overall incidence for the entire period was $5.8(95 \% \mathrm{Cl}$ : 4.9-6.6) per 100,000. Incidence was 2 to 7 times higher in females compared to males in the different age classes (5.9 times higher on average), and increased progressively with age, culminating at 19.6 per 100,000 in females aged $65-74$ years, with a slight decline thereafter to 15.9 per 100,000 among females aged 75 years and older. The incidence increased with calendar time over the 40-year period ( $p=0.005$, figure). There was no apparent seasonality in the incidence of pSS, with similar number of cases diagnosed during all four seasons. There was no difference in mortality in the pSS cohort compared to expected (standardized mortality ratio $1.15,95 \% \mathrm{Cl} 0.86-1.50$ ).

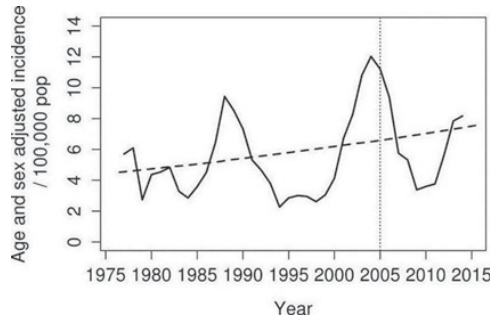

Conclusions: The average annual incidence of pSS in this population basedcohort was 5.8/100,000, with a progressive increase over the 40 years of the study. Overall survival of pSS patients was not different from the general population. References:

[1] Nannini C et al. BMJ Open. 2013;3:e003569.

Disclosure of Interest: None declared

DOI: 10.1136/annrheumdis-2017-eular.1178

\section{SAT0287 TH1, TH2 AND TH17 LYMPHOID SUBPOPULATIONS IN PRIMARY ANTIPHOSPHOLIPID SYNDROME}

G. Medina ${ }^{1}$, O.I. Florez-Durante ${ }^{2}$, L. Montiel Cervantes ${ }^{3}$, R. Molina-Aguilar ${ }^{3}$ E. Reyes-Maldonado ${ }^{2}$, L.J. Jara ${ }^{4} .{ }^{1}$ Clinical Research Unit, Instituto Mexicano del Seguro Social, Hospital de Especialidades Centro Médico la Raza; ${ }^{2}$ Escuela Nacional de Ciencias Biológicas, Instituto Politécnico Nacional; ${ }^{3} \mathrm{Hematology}$ Laboratory; ${ }^{4}$ Direction of Education and Research, Instituto Mexicano del Seguro Social, Hospital de Especialidades Centro Médico la Raza, Mexico City, Mexico

Background: Primary antiphospholipid antibody syndrome (PAPS) is characterized by thrombosis at different levels and maternal fetal complications in the presence of antiphospholipid antibodies (aPL). Lymphoid subpopulations and cellular immune responses have not been fully studied.

Objectives: To analyze the lymphoid subpopulations, Th1, Th2 and Th17 immune response in patients with PAPS and long term evolution.
Methods: Patients with PAPS, $>18$ years of age, of both sexes and a group of healthy blood donors matched for age and sex were included. All patients were receiving oral anticoagulants (Coumadin type). No patient had a recent episode of thrombosis or other manifestation of APS at the time of the study. Peripheral blood was obtained and lymphoid subpopulations were determined by flow cytometry in order to identified with specific immunological markers for Treg cells: CD4+/CD25+/FoxP3+ and CD8+/CD25+/FoxP3+. The dendritic cells analyzed were: Type 1: Lin1- HLA-DR+/CD11C+; Type 2: Lin-HLA-DR+/CD123+; B lymphocytes with antiCD19-APC; Monocytes with anti-CD14-PE; NK: CD3/CD16+56+ and NKT: CD3+/CD16+56+ lymphocytes. Th1 cells were identified by IFN-g ${ }^{+}$positivity; Th2: positivity for IL-4+; Th17: positivity for IL-17+. Parametric statistics and Mann-Whitney U-test were used.

Results:

\begin{tabular}{lccc}
\hline & PAPS $($ cells $/ \mu \mathrm{L})$ & Healthy controls $($ cells $/ \mu \mathrm{L})$ & $\mathrm{p}$ \\
\hline Total leukocytes & 5655 & 7000 & $<0.05^{\star}$ \\
Lymphocites & 1800 & 2085 & $\mathrm{NS}$ \\
Total CD3 & 1033 & 1338 & $\mathrm{NS}$ \\
Total CD4 & 701 & 775 & $\mathrm{NS}$ \\
Total CD8 & 258 & 441 & $<0.05^{\star}$ \\
Monocytes & 213 & 219 & $\mathrm{NS}$ \\
B lymphocites & 41 & 86 & $\mathrm{NS}$ \\
NK & 118 & 177 & $\mathrm{NS}$ \\
NKT & 33 & 45 & $\mathrm{NS}$ \\
iNKT & 9 & 20 & $<0.005^{\star \star}$ \\
T $\gamma$ Lymphocites & 34 & 45 & $\mathrm{NS}$ \\
LTreg 4 Lymphocites & 23 & 13 & $\mathrm{NS}$ \\
Treg 8 Lymphocytes & 26 & 13 & $\mathrm{NS}$ \\
DC1 & 2 & 4 & $<0.005^{\star \star}$ \\
DC2 & 1 & 5 & $<0.0005^{\star \star \star}$ \\
\hline
\end{tabular}

A total of 39 patients with PAPS were included, age: $51.9 \pm 12.8$, evolution time: $12.8 \pm 8.9$ years and 35 healthy controls. In patients with PAPS there was a decrease in the total CD8 count $(p<0.05)$ in iNKT $(p<0.005)$, DC1 $(p<0.005)$ and DC2 $(p<0.0005)$ cells compared to the control group (Table 1). We found significant decrease in Th1, Th2 and Th17 cytokines basal and after activation compared to healthy controls.

Conclusions: This study shows profound alterations in innate and adaptive immunity in patients with long-term PAPS, characterized by a decrease in certain lymphocyte subpopulations, with consequent functional alteration. These abnormalities can become new therapeutic targets in order to restore immune imbalance. Our findings may explain in part, the development of thrombosis and other complications, despite treatment with oral anticoagulants in PAPS patients with long term disease evolution.

\section{References:}

[1] Dal Ben ER, do Prado CH, Baptista TS, Bauer ME, Staub HL. Decreased levels of circulating CD4+CD25+Foxp3+regulatory $T$ cells in patients with primary antiphospholipid syndrome. J Clin Immunol 2013; 33: 876-879.

[2] Xiao J, Zhu F, Liu X, Xiong J.Th1/Th2/Th17/Treg expression in cultured PBMCs with antiphospholipid antibodies.Mol Med Rep. 2012;6(5):1035-9.

[3] Jakiela B, Iwaniec T, Plutecka H, Celinska-Lowenhoff M, Dziedzina S, Musial J. Signs of impaired immunoregulation and enhanced effector T-cell responses in the primary antiphospholipid syndrome. Lupus. 2016; 25(4):389-98.

Disclosure of Interest: None declared

DOI: 10.1136/annrheumdis-2017-eular.6222

\section{SAT0288 QUALITY OF LIFE IN PATIENTS WITH SYSTEMIC LUPUS ERIYTHEMATOSUS}

G. Voicu $^{1}$, L. Groseanu ${ }^{1}$, M. Abobului ${ }^{1}$, V. Vlad ${ }^{1}$, F. Berghea ${ }^{1}$, D. Predeteanu ${ }^{1}$, V. Bojinca ${ }^{2}$, I. Saulescu ${ }^{2}$, A. Borangiu ${ }^{2}$, S. Daia ${ }^{2}$, D. Mazilu ${ }^{2}$, A. Balanescu ${ }^{2}$, C. Constantinescu ${ }^{2}$, D. Opris ${ }^{2}$, R. Ionescu ${ }^{2} .{ }^{1}$ Rheumatolgy; ${ }^{2}$ internal medicine and Rheumatolgy, Sfanta Maria Clinic Hospital, Bucharest, Romania

Background: The Quality of life (QoL) in patients with SLE is deeply affected by multiple factors including psychological and physical factors, the functional status and the general perception over health.

Objectives: Evaluation of Quality of life and the perceptions over the disease in patients with SLE.functional status and the general perception over health.

Methods: This is a 10 month prospective, cross-sectional study performed on 52 patients hospitalized diagnosed with SLE according to SLICC 2012 criteria. There were evaluated demographic data, organs manifestations, disease activity scores (SLEDAI, SLICC) and treatment. To evaluate the QoL several questionnaires were performed: Health Assessment Questionnaire (HAQ), EuroQol five dimensions questionnaire (EQ5d), Illness Perception Questionnaire and SF-36.

Results: All patients were women with the mean age 49 years with a mean durations of illness 136 month (14). The mean SLEDAI - 5,52 $(5,37)$ and SLICC $-2,25(1,71)$.

The mean $H A Q$ value was $0,83(0,81)$. The $H A Q$ score doesn't correlate with SLICC $(p=0,461)$ and with SLEDAI $(p=0,172)$, but it was correlated with the neuropsychiatric manifestations. So the psychiatric affectation influences performing the usually daily activities $(p=0,005)$.

When assessing IIIness Perception Questionnaire, patients considered their life seriously affected by pain, mean value $6,3(2,6), 72 \%$ consider the illness will be 
present for the rest of their lives, $90 \%$ appreciate that treatment is improving the QoL mean $9,3(1,3), 68 \%$ consider to have many severe symptoms, more than $80 \%$ patients consider themselves preoccupied for their illness, more than $70 \%$ are emotionally affected by their illness and $48 \%$ consider stress being the main determinant factor of their illness.

Questionnaire EQ-5d: $10 \%$ must stay in bed due to the disease, $8,33 \%$ can not wash or dress themselves, $12,5 \%$ are unable to perform usual daily activities, $62 \%$ experience pain or a moderate uncomfortable state and $65 \%$ are worried and or depressed. Cardio-vascular manifestations are correlated with diminished mobility $(p=0,002)$ leading to deficiency in self care $(p=0,002)$ also correlated with renal $(p=0,034)$ and psychiatric $(p=0,022)$ manifestations. Psychiatric manifestations also affect usual daily activities $(p=0,05)$. Age is correlated with pain and uncomfortable state $(p=0,004)$ but the responses to this item of EQ5D are not correlated with organ affectation, biological modification or disease activity scores. The presence of cutaneous manifestations is correlated with anxiety and depression $(p=0,02)$.

Based on SF36 when calculating the mean value on each category was observed all the 8 categories affected - smallest values at physical role $(37,32)$ and general health $(34,21)$, less influenced part being the social function $(51,32)$.None of this items of SF 36 is correlated with SLICC or SLEDAI.

Conclusions: SLEDAI as well as SLICC or specific organ involvement give no indications over the patients QoL, which must be assessed by performing HAQ and SF 36.

[1] Lupus. 2016 Mar 16. pii: 0961203316638934 . [Epub ahead of print]Gender differences in systemic lupus erythematosus concerning anxiety, depression and quality of life.Macêdo EA1, Appenzeller S2, Costallat LT2.

Disclosure of Interest: None declared

DOI: 10.1136/annrheumdis-2017-eular.5316

\section{SAT0289 CARDIOVASCULAR RISK STATUS MODIFICATION IN SLE PATIENTS BY MEANS OF CAROTID ULTRASOUND EXAMINATION}

H. Sánchez-Pérez, B. Tejera-Segura, I. Ferraz-Amaro. Rheumatology, Hospital Universitario de Canarias, la Laguna, Spain

Background: Systemic lupus erythematosus (SLE) is associated with a higher cardiovascular risk (CVR), at least doubled when compared with the general population, as well as an increased prevalence of atherosclerosis, which occurs prematurely and independently of traditional CVR factors. The presence of atheromatous plaques documented with carotid ultrasound (US) examination implies a change in the patient's CVR category to "very high risk", according to the "European Guidelines on cardiovascular disease prevention in clinical practice, 2016". General guidelines do not take into account the specific increase in CVR risk that it's present in patients with autoimmune diseases.

Objectives: To examine if the use of carotid US leads to a more accurate CVR classification in SLE patients, and in that way to a better CVR management. Methods: Cross-sectional study that emcompassed 102 SLE patients currently being followed in our Rheumatology Department. Demographic variables, the presence of CVR factors and previous/current CV events, disease duration and drug use were analysed; lipid profile was assessed using blood tests, as well as carotid intima media thickness and the presence of carotid plaques using US scan. The current CVR status was calculated using the SCORE classification (low risk $<1 \%$, moderate risk $<5 \%$, high risk $<10 \%$ and very high risk $>10 \%$ ).

Results: $95 \%$ of our patients were women,mean age was $51.6+/$ - years,median disease duration was 16 years (IQR 9-28). 35.29\% of patients presented with at least one CVR factor before the SLE diagnosis, and $61.76 \%$ at the time of the research (19.60\% current smokers, 39.21\% high blood pressure (HBP),33.33\% dyslipidemia, $7.84 \%$ type II diabetes. A CV event was diagnosed in $11.76 \%$ of our patients throughout their SLE disease. According to the SCORE classification, 55 of them had a low CVR (53.92\%),26 moderate CVR (25.49\%),6 high CVR $(5.88 \%)$ and 15 of them very highCVR $(14.70 \%)$. Carotid plaques were found in 28 patients $(27.45 \%): 23$ of them had never had a CV event $(22.54 \%), 21.42 \%$ of them were current smokers, $64.28 \%$ had HBP, $53.57 \%$ dyslipidemia and $17.86 \%$ type II diabetes. After adjustment of the SCORE classification with the presence of carotid plaques $16.36 \%$ of patients from the "low CVR" category would be re-classified has having "very high CVR", as well as $30.76 \%$ from the "moderate CVR" category and $50 \%$ from the "high CVR" category. Of note, 8 patients within the "very high CVR" category also presented carotid plaques. Out of this patients with atheroma plaque, 13 were not using statins, and in 2 of them it would have been mandatory in order to achieve the LDL $<70 \mathrm{mg} / \mathrm{dl}$ target. In 2 of the 15 patients that were indeed under statins this target was not achieved. $60.71 \%$ of the atheromatous patients were under hydroxicloroquine use and $17.64 \%$ of them used steroids as a regular basis (33.33\% of them within the medium range dose) Conclusions: More than half of our SLE patients presented with at least 1 CVR factor. Around $15 \%$ of patients were categorised as "very high CVR" according to the SCORE classification. The presence of carotid plaque was found in $27.45 \%$ of our patients, and $82 \%$ of them hadn't suffered any CV event. The use of carotid US examination allowed us to re-classify 20 patients $(19.60 \%)$ in the "very high risk" category according to the SCORE classification. The need for a change in CVR management (ie., change in statin regime)was demonstrated in 4 of this 20 "re-classified" patients.
Disclosure of Interest: None declared

DOI: 10.1136/annrheumdis-2017-eular.6632

\section{SAT0290 OSTEONECROSIS IS THE MOST COMMON ORGAN DAMAGE AND IS ASSOCIATED WITH CUMULATIVE STEROID DOSAGE AS WELL AS LUPUS NEPHRITIS ITSELF IN PATIENTS WITH SLE}

H.H. Kwon ${ }^{1}$, S. Won ${ }^{2}$, Y.B. Joo ${ }^{3}$, M.J. Kim ${ }^{1}$, H.Y. Kim ${ }^{1}$, G.Y. Ahn ${ }^{1}$, S.-C. Bae ${ }^{1}$ ${ }^{1}$ Hanyang University Hospital for Rheumatic Diseases; ${ }^{2}$ Clinical Research Center for Rheumatoid Arthritis (CRCRA), Seoul; ${ }^{3}$ St Vincent's Hospital, Suwon, Korea, Republic Of

Background: In patients with SLE, musculoskeletal system is common organ system involved cumulatively over time. Osteonecrosis (ON) is common in musculoskeletal damage accrual and often disabling. Steroid use has been a risk factor in the development of ON, but SLE itself has also been suggested to have a role. It is important to understand factors related to the development of ON.

Objectives: We investigated the overall profile of organ damage accrual, particularly musculoskeletal damage and focused on factors associated with ON in a single Asian cohort.

Methods: Patients with SLE who met American College of Rheumatology (ACR) criteria were enrolled and followed from 1998 to 2014 in the Hanyang BAE Lupus cohort. Systemic Lupus International Collaborating Clinics/American College of Rheumatology Damage Index (SDI) was measured annually. ON was confirmed by X-ray, bone scan or MRI. Patients with ON was compared to controls without ON from the same cohort for clinical, laboratory, and therapeutic factors. Univariate logistic regression followed by multivariate logistic regression were used to determine risk factors for ON.

Results: We recruited 1,219 SLE patients. The most common type of organ damage was musculoskeletal (205 patients, 16.8\%). ON was the most common subtype (133 patients, $10.9 \%$ ) of the musculoskeletal damage. The mean time from diagnosis of SLE to development of ON was $4.97 \pm 4.15(0.17 \sim 20.25)$ years. One hundred twenty-six patients were eligible for identifying distribution of ON. Overall, 291 joints were affected by ON and mean number of ON joint per patients with ON was $2.3 \pm 1.23(1 \sim 9)$. The percentage of $>2$ joint involvement was $24.6 \%$. The hip (femoral head) was the most frequently involved joint $(65.6 \%)$, followed by the knee (distal femur, proximal tibia, proximal fibula, patella: $20.6 \%$ ), shoulder (humeral head: $5.2 \%$ ) and ankle (distal tibia, distal fibula, talus: $5.2 \%$ ).

In univariate analysis, age at diagnosis, follow up duration, accumulative number of ACR criteria, serositis, renal disorder \& neurologic disorder (based on ACR criteria), cyclophosphamide \& mycophenolate use, and cumulative steroid dosage were significantly associated with $\mathrm{ON}$. In multivariate analysis, renal disorder $(p=0.0109)$, and cumulative steroid usage $(p=0.0131)$ were significantly associated with $\mathrm{ON}$.

To evaluate the effect of cumulative steroid usage on ON, age, sex and disease duration matched $\mathrm{ON}$ cases and controls (1:4, a total of 665 patients) were selected from the same cohort. Total cumulative steroid dosage was divided into 4 groups by grams $(>0 \& \leq 5 \mathrm{~g},>5 \mathrm{~g} \& \leq 10 \mathrm{~g},>10 \mathrm{~g} \& \leq 20 \mathrm{~g}$, and $>20 \mathrm{~g})$. More than $10 \mathrm{~g}$ group of cumulative steroid use was significantly associated with ON. And then we stratified 530 patients according to biopsy proven lupus nephritis (class III to V) and cumulative steroid dose (10g cut-off), and tested by Chi-square test and logistic regression. ON was significantly higher in the group with lupus nephritis within $>10 \mathrm{~g}$ group of total cumulative steroid.

\begin{tabular}{ccc}
\multicolumn{1}{c}{ Table 1. Total cumulative steroid dose as risk factor for ON } & \\
\hline Total cumulative steroid dose(g) & $\mathrm{OR}(95 \% \mathrm{Cl})$ & p-value \\
\hline$>0$ \& $\leq 5$ & 1.00 & \\
$>5 \& \leq 10$ & $2.00(0.91-4.39)$ & 0.0844 \\
$>10 \& \leq 20$ & $2.34(1.19-4.58)$ & 0.0133 \\
$>20$ & $2.96(1.60-5.47)$ & 0.0005 \\
\hline
\end{tabular}

Table 2-1. Association between Osteonecrosis and lupus nephritis based on total eumulative steroid $10 \mathrm{~g}$

\begin{tabular}{ccccc}
\hline Total cumulative steroid dose $>10 \mathrm{~g}$ (371 patients) & & & \\
\hline & Total & ON $(+)$ & ON $(-)$ & p-value \\
\cline { 2 - 5 } & 196 & $58(29.59)$ & $138(70.41)$ & 0.0018 \\
Lupus nephritis( $(+)$ & 175 & $27(15.43)$ & $148(51.75)$ & \\
Lupus nephritis $(-)$ & & & &
\end{tabular}

Table 2-2. Association between Osteonecrosis and lupus nephritis based on total cumulative steroid $10 \mathrm{~g}$

\begin{tabular}{lcc}
\hline & $O R(95 \% \mathrm{Cl})$ & $p$-value \\
\hline Steroid dose 10 \& Lupus nephritis(-) & 1.00 & \\
Steroid dose $>10$ \& Lupus nephritis(-) & $1.58(0.81-3.10)$ & 0.1826 \\
Steroid dose 10 \& Lupus nephritis $(+)$ & $2.48(1.02-5.99)$ & 0.0441 \\
Steroid dose $>10$ \& Lupus nephritis $(+)$ & $3.64(1.97-6.75)$ & $<0.0001$ \\
\hline
\end{tabular}

Conclusions: ON was the most frequent damage in lupus patients. We confirmed again cumulative steroid dosage as a risk factor for the development of ON. In addition, lupus nephritis itself was suggested as an independent risk factor for ON

Disclosure of Interest: None declared

DOI: 10.1136/annrheumdis-2017-eular.4547 